\title{
Evaluation of two-dimensional electronic portal imaging device using integrated images during volumetric modulated arc therapy for prostate cancer
}

\author{
Shoki Inui ${ }^{1,2}$, Yoshihiro Ueda ${ }^{1}$, Shunsuke Ono ${ }^{1}$, Shingo Ohira ${ }^{1}$, Masaru Isono ${ }^{1}$, Yuya Nitta ${ }^{1}$, Hikari Ueda ${ }^{1}$, \\ Masayoshi Miyazaki ${ }^{1}$, Masahiko Koizumi ${ }^{2}$, Teruki Teshima ${ }^{1}$ \\ ${ }^{1}$ Department of Radiation Oncology, Osaka International Cancer Institute, Osaka, Japan \\ ${ }^{2}$ Department of Medical Physics and Engineering, Osaka University Graduate School of Medicine, Suita, Japan
}

\begin{abstract}
Background: The aim of the study was to evaluate analysis criteria for the identification of the presence of rectal gas during volumetric modulated arc therapy (VMAT) for prostate cancer patients by using electronic portal imaging device (EPID)-based in vivo dosimetry (IVD).

Materials and methods: All measurements were performed by determining the cumulative EPID images in an integrated acquisition mode and analyzed using PerFRACTION commercial software. Systematic setup errors were simulated by moving the anthropomorphic phantom in each translational and rotational direction. The inhomogeneity regions were also simulated by the I'mRT phantom attached to the Quasar phantom. The presence of small and large air cavities $\left(12\right.$ and $\left.48 \mathrm{~cm}^{3}\right)$ was controlled by moving the Quasar phantom in several timings during VMAT. Sixteen prostate cancer patients received EPID-based IVD during VMAT.

Results: In the phantom study, no systematic setup error was detected in the range that can happen in clinical $<5-\mathrm{mm}$ and $<3$ degree). The pass rate of $2 \%$ dose difference (DD2\%) in small and large air cavities was $98.74 \%$ and $79.05 \%$, respectively, in the appearance of the air cavity after irradiation three quarter times. In the clinical study, some fractions caused a sharp decline in the DD2\% pass rate. The proportion for DD2\% < $90 \%$ was $13.4 \%$ of all fractions. Rectal gas was confirmed in $11.0 \%$ of fractions by acquiring kilo-voltage $\mathrm{X}$-ray images after the treatment.

Conclusions: Our results suggest that analysis criteria of $2 \%$ dose difference in EPID-based IVD was a suitable method for identification of rectal gas during VMAT for prostate cancer patients.

Key words: in vivo dosimetry (IVD); electronic portal imaging device (EPID); prostate cancer; rectal gas; patient-specific quality assurance (QA)

Rep Pract Oncol Radiother 2021;26(2):281-290
\end{abstract}

\section{Introduction}

High-precision radiation therapy, such as intensity modulated radiation therapy (IMRT) and volumetric modulated arc therapy (VMAT), can be used to create a steep dose gradient and com- plement dose distribution $[1,2]$. These techniques enable the target dose to be increased and the organ at risk (OAR) dose to be decreased. Treatment of prostate cancer patients has often used IMRT and VMAT, resulting in better tumor control and reduced toxic effects in the bladder and rectum [3-5].

Address for correspondence: Yoshihiro Ueda, Department of Radiation Oncology, Osaka International Cancer Institute, 3-1-69 Otemae, Chuou-ku, Osaka, 537-8567, Japan, fax: +81-6-6981-3000; e-mail: ueda-yo@mc.pref.osaka.jp 
However, a steeper dose gradient, more complex dose distribution, and a smaller planning target volume (PTV) margin may cause geometrical uncertainties, such as interfractional and intrafractional setup variation and organ movement, that displace the target from the treatment field. Image-guided radiation therapy (IGRT) is used to aid precise dose delivery to the target. This method enables the interfractional setup variation to be reduced as much as possible.

Intrafractional organ movement in prostate cancer treatment has been studied over the past 20 years $[6,7]$. Some studies have used magnetic resonance imaging (MRI) to monitor intrafractional prostate and rectum movement in real time during treatment [6-11]. These studies suggest that rectal gas causes a distended rectum, thereby displacing the prostate. This indicates that rectal gas can be used as an indicator of intrafractional rectum and prostate movement. Moreover, rectal gas causes an increase in the inhomogeneity of the density of materials in the beam path, altering the dose distribution and causing the appearance of hot spot in the rectal wall and prostate. It is therefore important to determine the presence of rectal gas during treatment.

Several studies reported that an electronic portal imaging device (EPID) had usefulness in the dose verifications for pre-treatment $[12,13]$. EPID-based in vivo dosimetry (IVD) is one of the patient-specific quality assurance (QA) methods and it is widely used to detect major treatment errors during radiation therapy [14-22]. EPID-based IVD is also a real-time verification system that is easy to use and does not require additional setup time for IMRT and VMAT. This system ensures safety and accuracy on a daily basis by measuring and analyzing the beam penetrating the patient's body during treatment in the integrated acquisition mode. Such a verification system is an additional tool that can help reveal major treatment errors such as intrafractional patient and organ movement during IMRT and VMAT. However, little has been done to identify the presence of rectal gas using EPID-based IVD in VMAT for prostate cancer patients. We hypothesized that inhomogeneity regions such as those with rectal gas can be changed owing to the beam passing through the patient's body and detected using EPID-based IVD. Hence, the aim of this study was to evaluate analysis cri- teria for the identification of the presence of rectal gas when EPID-based IVD is used in VMAT for prostate cancer patients. A phantom study identified the magnitude of error in EPID-based IVD when setup error and rectal gas in the range that can happen in a clinical study occurs. In a clinical study, the feasibility of using EPID-based IVD to detect rectal gas was assessed based on the results of the phantom study.

\section{Material and methods}

This research consists of three parts: two phantom studies and one clinical study. Settings that are common among the three studies are described in the following section, while settings specific to each study are specified in the section after that.

\section{Common settings}

In the phantom and clinical studies, all computed tomography (CT) scans were performed using a GE Dual Energy instrument (64 slices, General Electric Co., Waukesha, WI). The parameters of the CT images were $2.0-\mathrm{mm}$ slice thickness and $500-\mathrm{mm}$ field of view with dimensions of $512 \times 512$ pixels. All the CT images were transferred to an Eclipse planning system (version 13.7.14, Varian Medical Systems, Palo Alto, CA). In Eclipse, all plans were calculated with the analytical anisotropic algorithm for dose calculation with inhomogeneity corrections, and exported to the PerFRACTION software (version 1.7.3, Sun Nuclear Corporation, Melbourne, FL) as DICOM files.

PerFRACTION, which uses GPU-accelerated convolution/superposition algorithm (Sun Nuclear Dose Calculator) and is independent of the Eclipse planning system, is a commercial software for pre- and on-treatment patient-specific QA by using the EPID images. This system was used for the on-treatment patient-specific QA (Fraction N) in our study. The feature of PerFRACTION is automatic retrieval of the acquired new EPID images and comparison of these images against the baseline images through various user-defined tests including gamma analysis, percentage dose difference (DD), and distance-to-agreement (DTA) for any criteria and threshold. The beam model of PerFRACTION is created by Sun Nuclear Corporation following the data of percent depth dose, 
output factor, and profile in reference condition and VMAT planning often used in our institute.

EPID-based IVD was performed using a linear accelerator, TrueBeam STx (Varian Medical Systems) equipped with a gantry-mounted on-board imager and an EPID, aS1200 PortalVision image detector (Varian Medical Systems). The square pixels of the EPID had a side length of $0.34 \mathrm{~mm}$, yielding a total area of approximately $40 \times 40 \mathrm{~cm}^{2}$ $(1190 \times 1190$ pixels $)$. All EPID images were obtained in the integrated acquisition mode, and a source-to-imager distance of $160 \mathrm{~cm}$ was used for both the stationary and rotational settings. The linear accelerator was equipped with high definition multileaf collimators (MLCs), the widths of which were $2.5 \mathrm{~mm}$ for the first 32 leaves from the central point and $5 \mathrm{~mm}$ for the rest.

\section{Specific settings}

\section{First phantom study}

To assess the detectability of a setup error in EPID-based IVD, an anthropomorphic phantom (Kyoto Kagaku, Japan) was used (Fig. 1A). In CT images of the anthropomorphic phantom, the prostate was contoured as the target volume, while the rectum, bladder, and small and large bowels were contoured as OARs. A single-arc VMAT plan using 2 Gy fractions with 6-MV photons was created for target contouring. The plans were op- timized to deliver the mean of the prescribed dose to the PTV.

To acquire baseline measurements, the anthropomorphic phantom was correctly set up by using cone-beam computed tomography (CBCT) image guidance and irradiated while the cumulative EPID images were acquired. Systematic setup errors were simulated by shifting the phantom by $1,3,5$, and 10 - $\mathrm{mm}$ in the anterior-posterior (AP), superior-inferior (SI), and left-right (LR) directions. Angular setup errors were also simulated by shifting the phantom by $1^{\circ}$ and $3^{\circ}$ in the pitch, roll, and yaw rotations. The EPID images were captured for each condition.

\section{Second phantom study}

To assess the detectability of inhomogeneity regions in the rectum in EPID-based IVD, an I'mRT phantom comprised of a body phantom (IBA Dosimetry, Schwarzenbruck, Germany) was used (Fig. 1B). The mock prostate structure set downloaded from AAPM TG119 was registered on CT images of the I'mRT phantom with MIM Maestro (MIM Software Inc., OH, USA). A single-arc VMAT plan using 2 Gy fractions with 6-MV photons was optimized to deliver the mean of the prescribed dose to the PTV.

To acquire baseline measurements, the I'mRT phantom was correctly set up by using CBCT im-

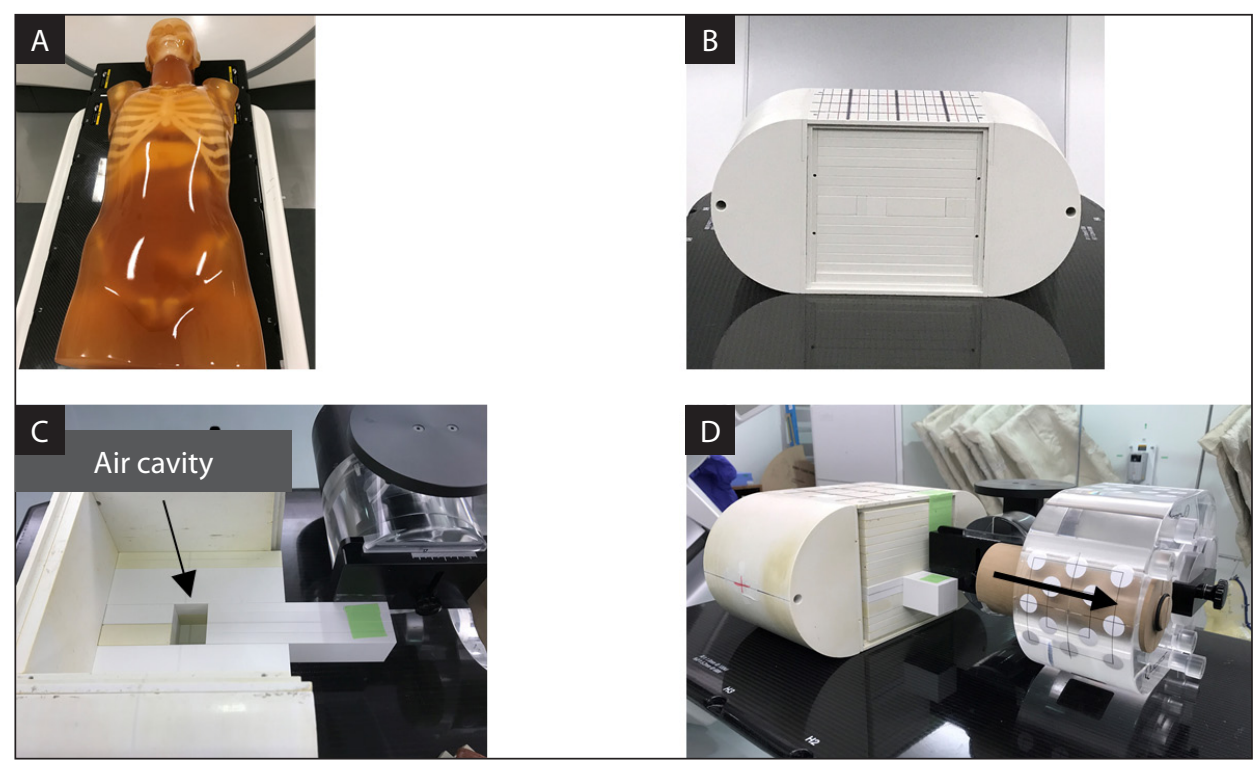

Figure 1. A. The anthropomorphic phantom for the verification of systematic setup error. B. The I'mRT phantom for the verification of inhomogeneity region. $\mathbf{C}$. The position of the air cavity in the I'mRT phantom. $\mathbf{D}$. The programmable motion platform stuck on the I'mRT phantom and made up the air cavity after several times during irradiation 
age guidance and irradiated while the cumulative EPID images were acquired. Multiple-size air cavities which simulated rectal gas were created in the I'mRT phantom. We prepared the air cavities with diameters of 2 and $4 \mathrm{~cm}$ because the mean rectal diameter in clinical study was about $3 \mathrm{~cm}$. Each air cavity, with volume of either $12(2 \times 2 \times 3) \mathrm{cm}^{3}$ or 48 $(4 \times 4 \times 3) \mathrm{cm}^{3}$ was created $1 \mathrm{~cm}$ below the middle of the I'mRT phantom (Fig. 1C).

Rectal gas may occur during irradiation, so we established a system controlling the time when gas is produced in the I'mRT phantom. The I'mRT phantom was attached next to a programmable motion platform (Quasar Respiratory Motion Platform; Modus Medical Devices Inc., London, ONT, Canada) (Fig. 1D). At the time when gas was scheduled to occur, some blocks attached to the programmable motion platform were pulled, and air cavities of volumes $12(2 \times 2 \times 3) \mathrm{cm}^{3}$ and $48(4 \times 4 \times 3) \mathrm{cm}^{3}$ were created $1 \mathrm{~cm}$ below the middle of the I'mRT phantom. The timing of when the air cavities occurred was controlled by moving the motion phantom during irradiation. Three timings were prepared and followed: at $25 \%\left(\mathrm{~T}_{25 \%}\right)$, $50 \%\left(\mathrm{~T}_{50 \%}\right)$, and $75 \%\left(\mathrm{~T}_{75 \%}\right)$ of the single-arc irradiation time. The I'mRT phantom with small and large air cavities were also measured during single-arc VMAT from beginning to end ( $\mathrm{T}_{0 \%}$ ). The EPID images were captured for each condition with I'mRT phantom with each air cavity.

\section{Clinical study}

From August 2017 to May 2018, 16 patients with histologically proven low-, intermediate- or high-risk localized prostate cancer underwent VMAT at the Osaka International Cancer Institute. The patients were aged 65 to 81 years (median: 77 years). All patients were required to empty their bladder and evacuate their bowels $1 \mathrm{~h}$ before the CT simulation. For the CT simulation, they were immobilized using the Vac-Lock Cushion (Civco Medical Solutions, Iowa, USA) for the pelvis region and they were given leg support and a pillow. After the immobilization devices were fixed, a treatment-planning CT scan was performed. If the rectum was deemed too large, the patients had to retake the scan after the rectum was emptied. After CT simulation, the target volumes and OARs (i.e., the rectum, bladder, and small and large bowels) were contoured by radiation oncologists following the recommendations in Reports 50 and 62 of the International Commission on Radiation Units [23, 24]. All the patients were treated with 6-MV photons using single-arc VMAT and received doses of $74 \mathrm{~Gy}$ in 37 fractions or $78 \mathrm{~Gy}$ in 39 fractions. The doses were prescribed at mean dose for the PTV.

All the patients received daily soft tissue registration, which was performed using $\mathrm{CBCT}$, and planning CT in a 6 degree of freedom couch. If the rectum was full of rectal gas near the prostate, the patient was encouraged to evacuate their bowels and registration was performed again. All the patients received VMAT while the cumulative EPID images were acquired in all the sessions. The EPID image in the first treatment session was defined as the baseline image. After treatment, all the patients were subjected to registration using a pair of orthogonal kilo-voltage X-ray images and DRR to determine the presence of rectal gas.

\section{Data analysis}

Two-dimensional (2D) analysis in PerFRACTION was performed using gamma and DD methods. Analysis criteria of $3 \% / 3 \mathrm{~mm}, 2 \% / 2 \mathrm{~mm}$, and $1 \% / 1 \mathrm{~mm}$, and $3 \%, 2 \%$, and $1 \%$, respectively, were used for the gamma and DD analysis above $10 \%$ of maximum signal threshold. The presence of rectal gas after treatment was confirmed via offline reviews (Varian Medical Systems).

\section{Results}

\section{Phantom study}

Single-arc VMAT was performed on the anthropomorphic phantom to assess the effect of systematic setup error on EPID-based IVD, and the results are shown in Table 1. The gamma analysis revealed $>95 \%$ in shifts of the AP and LR directions, and pitch, roll, and yaw rotations (Fig. 2). The pass rates of the DD were the same as those of the gamma analysis in the AP and LR directions of less than $5-\mathrm{mm}$ shift, and pitch, roll, and yaw rotations. In the SI direction, the pass rates for the DD of $1 \%$ in more than 3-mm shifts, and for the gamma of $1 \% / 1 \mathrm{~mm}$ and for the DD of $2 \%$ in more than $5-\mathrm{mm}$ shifts were less than $95 \%$.

Table 2 illustrates the effect of the presence of inhomogeneity regions on EPID-based IVD in I'mRT phantom with an air cavity. A larger air cavity resulted in decreased pass rates for the gamma 
Table 1. Systematic setup error simulated by shifting anthropomorphic phantom

\begin{tabular}{|c|c|c|c|c|c|c|c|}
\hline \multirow{2}{*}{ Direction } & \multirow{2}{*}{ Shift } & \multicolumn{3}{|c|}{ Gamma (\%) } & \multicolumn{3}{|c|}{ DD (\%) } \\
\hline & & $3 \% / 3 \mathrm{~mm}$ & $2 \% / 2 \mathrm{~mm}$ & $1 \% / 1 \mathrm{~mm}$ & $3 \%$ & $2 \%$ & $1 \%$ \\
\hline & No shift & 100.00 & 100.00 & 100.00 & 100.00 & 100.00 & 100.00 \\
\hline \multirow[t]{4}{*}{ AP } & $+1 \mathrm{~mm}$ & 99.99 & 99,98 & 99.98 & 99.98 & 99.98 & 99.95 \\
\hline & $+3 \mathrm{~mm}$ & 99.99 & 99.98 & 99.98 & 99.98 & 99.98 & 99.83 \\
\hline & $+5 \mathrm{~mm}$ & 99.99 & 99.98 & 99.96 & 99.98 & 99.98 & 97.05 \\
\hline & $+10 \mathrm{~mm}$ & 99.99 & 99.95 & 99.33 & 99.87 & 98.77 & 79.59 \\
\hline \multirow[t]{4}{*}{ SI } & $+1 \mathrm{~mm}$ & 99.99 & 99.98 & 99.56 & 99.93 & 98.85 & 96.49 \\
\hline & $+3 \mathrm{~mm}$ & 99.99 & 99.01 & 96.84 & 97.25 & 95.77 & 89.77 \\
\hline & $+5 \mathrm{~mm}$ & 99.98 & 97.73 & 94.80 & 95.29 & 93.08 & 80.31 \\
\hline & $+10 \mathrm{~mm}$ & 99.85 & 95.74 & 89.87 & 90.59 & 85.28 & 69.17 \\
\hline \multirow[t]{4}{*}{ LR } & $+1 \mathrm{~mm}$ & 99.99 & 99.98 & 99.98 & 99.99 & 99.98 & 99.97 \\
\hline & $+3 \mathrm{~mm}$ & 99.99 & 99.98 & 99.95 & 99.99 & 99.87 & 97.03 \\
\hline & $+5 \mathrm{~mm}$ & 99.99 & 99.98 & 99.65 & 99.84 & 98.63 & 94.46 \\
\hline & $+10 \mathrm{~mm}$ & 99.97 & 99.76 & 96.98 & 97.44 & 94.28 & 85.88 \\
\hline \multirow[t]{2}{*}{ Pitch } & $+1^{\circ}$ & 100.00 & 100.00 & 100.00 & 100.00 & 100.00 & 99.95 \\
\hline & $+3^{\circ}$ & 100.00 & 100.00 & 100.00 & 100.00 & 100.00 & 99.87 \\
\hline \multirow[t]{2}{*}{ Roll } & $+1^{\circ}$ & 100.00 & 100.00 & 100.00 & 100.00 & 100.00 & 99.86 \\
\hline & $+3^{\circ}$ & 100.00 & 100.00 & 100.00 & 100.00 & 100.00 & 99.77 \\
\hline \multirow[t]{2}{*}{ Yaw } & $+1^{\circ}$ & 100.00 & 100.00 & 100.00 & 100.00 & 100.00 & 99.21 \\
\hline & $+3^{\circ}$ & 100.00 & 100.00 & 100.00 & 100.00 & 100.00 & 99.17 \\
\hline
\end{tabular}

DD — dose difference; AP — anterior-posterior; SI — superior-inferior; LR — left-right

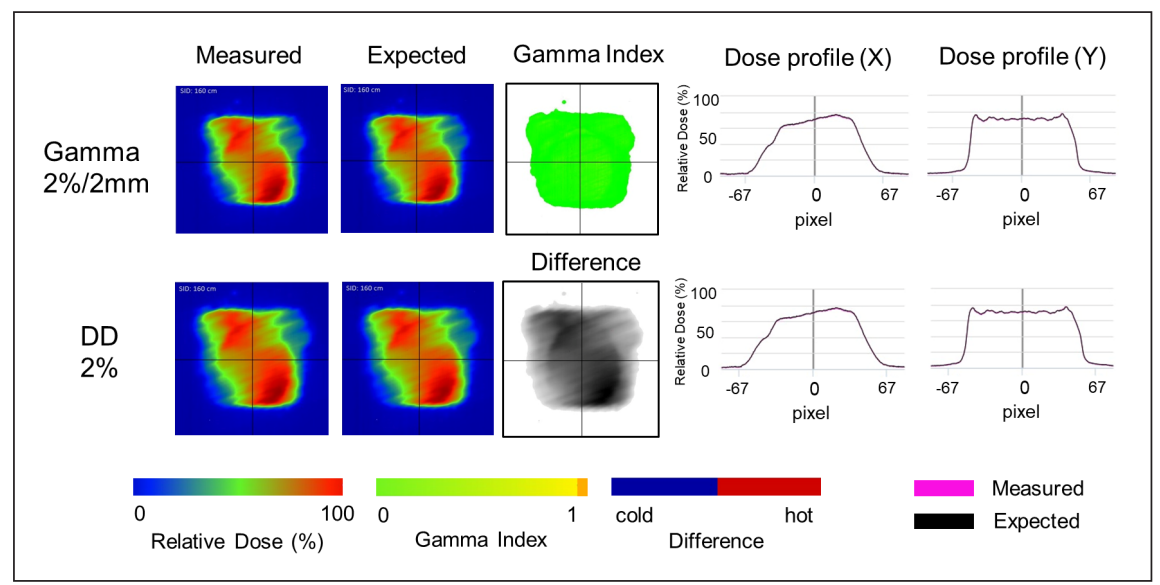

Figure 2. The comparison of the gamma and dose difference (DD) analysis in systematic setup error simulated by shifting the anthropomorphic phantom by $5 \mathrm{~mm}$ in the AP direction. The EPID images of expected dose are baseline. Dose profile $X$ and $Y$ are horizontal and vertical lines in the gamma index and difference

analysis of $1 \% / 1 \mathrm{~mm}$ and the DD of $3 \%, 2 \%$, and $1 \%$. A longer presence time for inhomogeneity regions during irradiation resulted in decreased pass rates for the gamma analysis of $1 \% / 1 \mathrm{~mm}$ and the DD of $3 \%$ of a large air cavity, and for the $\mathrm{DD}$ of $2 \%$ and $1 \%$ of large and small air cavities. However, all criteria of the gamma analysis were more than $90 \%$ pass rates for all phases in a small air cavity (Fig. 3).

Based on these results of phantom studies, we evaluated the results of the clinical study by using the $\mathrm{DD}$ of $2 \%(\mathrm{DD} 2 \%)$. At $\mathrm{T}_{75 \%}$, the air cavity appeared after $75 \%$ of the irradiation time and stayed for $25 \%$ of the irradiation time. Because the mean 
Table 2. Inhomogeneity regions simulated using the I'mRT phantom with air cavity

\begin{tabular}{|c|c|c|c|c|c|c|c|}
\hline Air cavity $\left[\mathrm{cm}^{3}\right]$ & Method & Criteria & $T_{0 \%}$ & $T_{25 \%}$ & $T_{50 \%}$ & $T_{75 \%}$ & $\mathbf{T}_{100 \%}$ \\
\hline \multirow{6}{*}{$12(2 \times 2 \times 3)$} & \multirow{3}{*}{ Gamma (\%) } & $3 \% / 3 \mathrm{~mm}$ & 99.98 & 99.98 & 99.99 & 99.99 & 100.00 \\
\hline & & $2 \% / 2 \mathrm{~mm}$ & 98.79 & 99.97 & 99.98 & 99.98 & 100.00 \\
\hline & & $1 \% / 1 \mathrm{~mm}$ & 91.55 & 92.29 & 97.96 & 98.61 & 100.00 \\
\hline & \multirow{3}{*}{ DD (\%) } & $3 \%$ & 92.46 & 92.78 & 99.98 & 99.99 & 100.00 \\
\hline & & $2 \%$ & 81.63 & 84.30 & 96.14 & 98.74 & 100.00 \\
\hline & & $1 \%$ & 61.32 & 74.90 & 79.37 & 86.09 & 100.00 \\
\hline \multirow{6}{*}{$48(4 \times 4 \times 3)$} & \multirow{3}{*}{ Gamma (\%) } & $3 \% / 3 \mathrm{~mm}$ & 99.73 & 99.82 & 99.99 & 99.99 & 100.00 \\
\hline & & $2 \% / 2 \mathrm{~mm}$ & 96.27 & 98.01 & 99.45 & 99.98 & 100.00 \\
\hline & & $1 \% / 1 \mathrm{~mm}$ & 80.91 & 87.98 & 93.80 & 96.90 & 100.00 \\
\hline & \multirow{3}{*}{$\mathrm{DD}(\%)$} & $3 \%$ & 69.76 & 80.52 & 83.19 & 88.89 & 100.00 \\
\hline & & $2 \%$ & 59.03 & 60.97 & 73.86 & 79.05 & 100.00 \\
\hline & & $1 \%$ & 40.44 & 46.98 & 55.11 & 56.50 & 100.00 \\
\hline
\end{tabular}

The appearance of air cavity was controlled by moving the motion phantom after quarter $\left(\mathrm{T}_{25 \%}\right)$, half $\left(\mathrm{T}_{50 \%}\right)$, and three to four times $\left(\mathrm{T}_{75 \%}\right)$ during one-arc VMAT. The I'mRT phantom with small and large air cavities during VMAT from beginning to end were also measured $\left(\mathrm{T}_{0 \%}\right)$. None of air cavity was also measured ( $\left.\mathrm{T}_{100 \%}\right)$; DD - dose difference

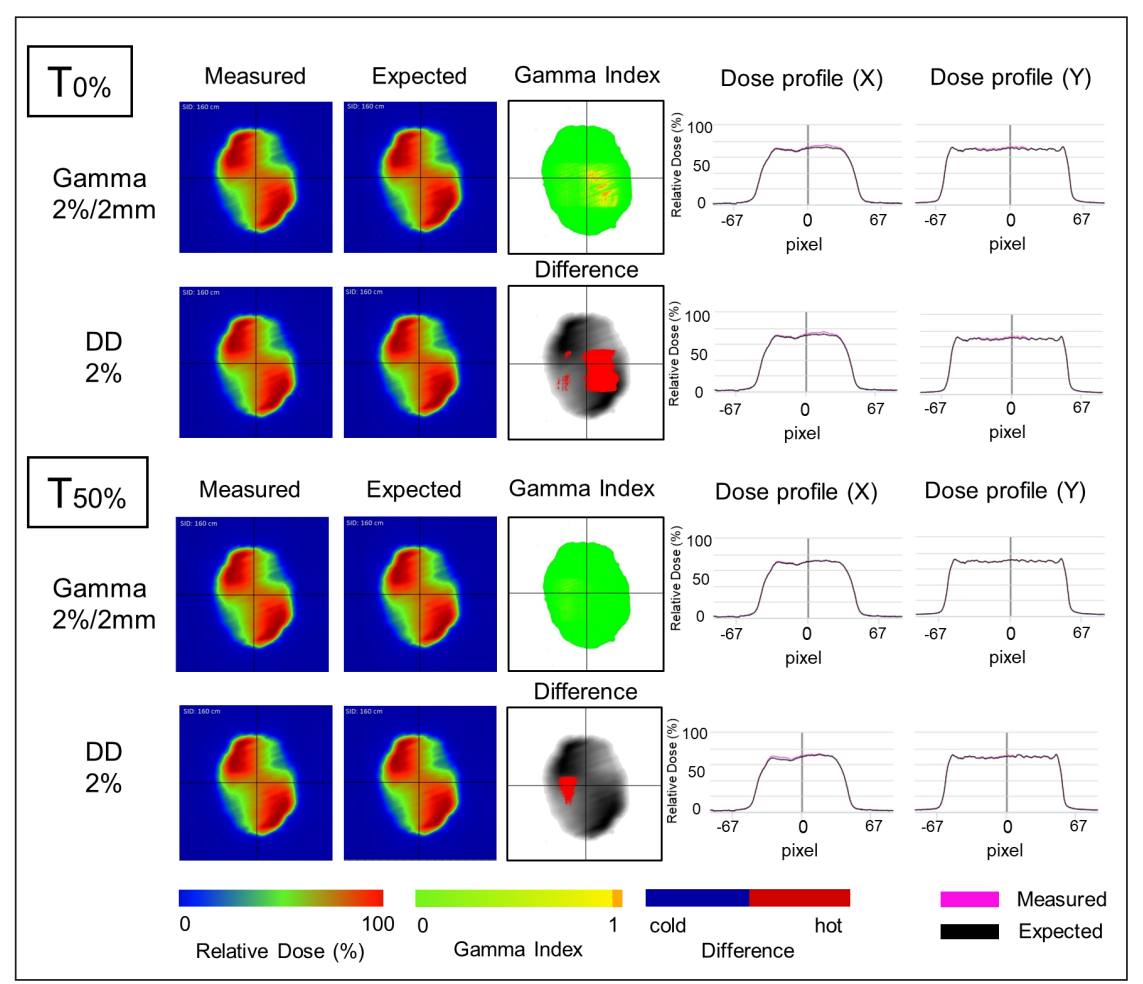

Figure 3. The comparison of the gamma and dose difference (DD) analysis in inhomogeneity regions simulated using the I'mRT phantom which had a small air cavity with or without the programmable motion platform movement. At $\mathrm{T}_{0 \%}$, the air cavity had stayed in the irradiation time from beginning to end. At $\mathrm{T}_{50 \%}$, the air cavity appeared after $50 \%$ of the irradiation time and had stayed for $50 \%$ of the irradiation time. The EPID images of expected dose are baseline. Dose profile $\mathrm{X}$ and $\mathrm{Y}$ are horizontal and vertical lines in the gamma index and difference

value of $\mathrm{DD} 2 \%$ at $\mathrm{T}_{75 \%}$ in the large and small air cavities was about $90 \%$, DD2\% $<90 \%$ was defined as the possibility of the rectum of being full of rectal gas during irradiation.

\section{Clinical study}

A total of 614 fractions from 16 patients were subjected to EPID-based IVD during VMAT. Among the total, 25 fractions were irretrievable 


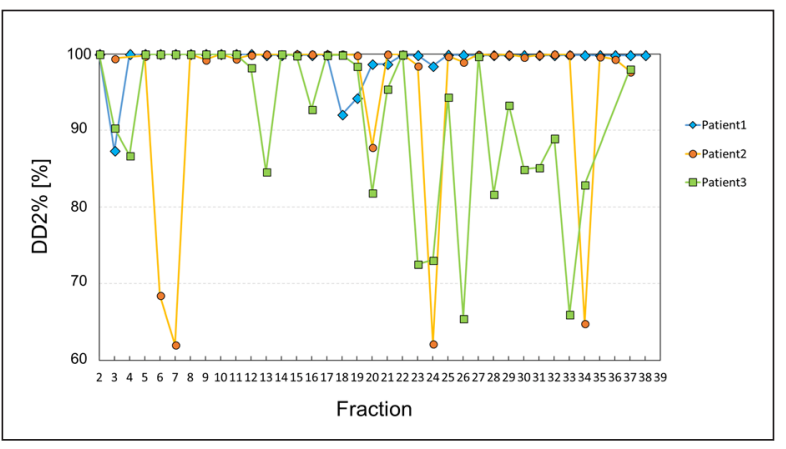

Figure 4. The daily transition of the pass rate in EPID-based IVD for three representative patients

because of a defect in data transmission. Figure 4 illustrates the daily variation in the pass rate in the EPID-based IVD for three representative patients. There was almost no change in the pass rate for some patients, but the pass rate changed for others in about one to three fractions. Figure 5 shows an example of a fraction with full rectal gas after clinical treatment displayed using PerFRACTION software. Some fractions could be used to confirm the location of rectal gas during treatment via the analysis of EPID images the same as Figure 5. Table 3 summarizes the analysis results of the clinical study for EPID-based IVD for prostate cancer. The results of nearly $90 \%$ of the fractions were DD2\%>90\% in all the patients. The average DD2\% value of the fractions with an empty rectum and a rectum full of rectal gas after irradiation were $96.12 \%$ and $86.13 \%$. Moreover, the proportion of $\mathrm{DD} 2 \%<90 \%$ indicated that there were more frac-
Table 3. Results of clinical study for EPID-based IVD for prostate cancer

\begin{tabular}{|l|c|}
\hline All patients ( $\mathrm{n}=\mathbf{1 6}, \mathbf{5 8 9}$ fractions) & Fraction (\%) \\
\hline $\mathrm{DD} 2 \% \geq 90 \%$ & $510(86.59)$ \\
\hline $\mathrm{DD} 2 \%<90 \%$ & $79(13.41)$ \\
\hline Empty rectum after irradiation & $524(88.96)$ \\
\hline Rectum full of rectal gas after irradiation & $65(11.04)$ \\
\hline
\end{tabular}

DD2\% - dose difference criteria $2 \%$

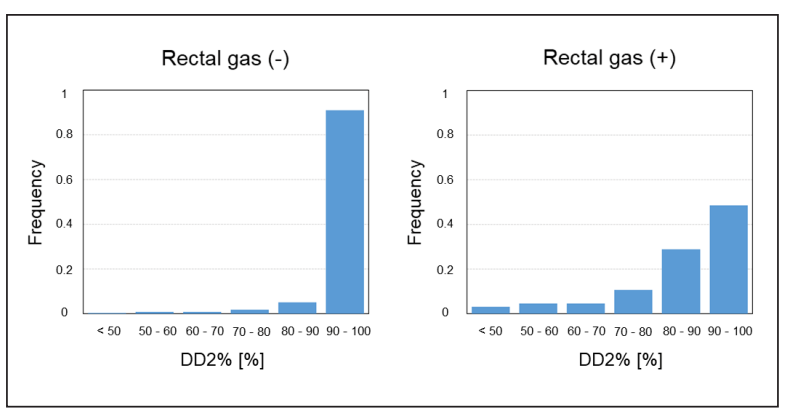

Figure 6. Dose difference distribution for the presence or absence of rectal gas after irradiation in a clinical study; DD2\% - dose difference criteria $2 \%$

tions with a rectum full of rectal gas than with an empty rectum (Fig. 6).

\section{Discussion}

Several studies have reported that EPID-based IVD can be used to identify major treatment errors such as intrafractional patient and organ movement [15-22]. However, these studies did not clearly

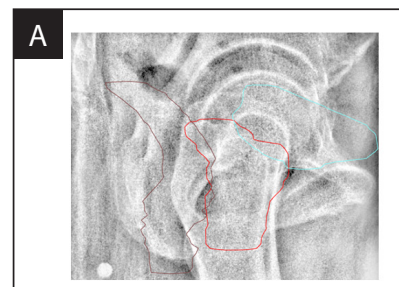

B

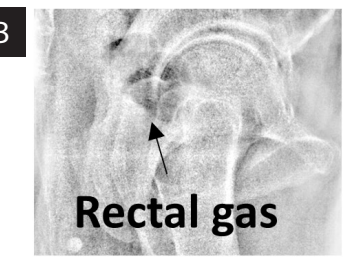

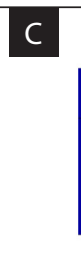

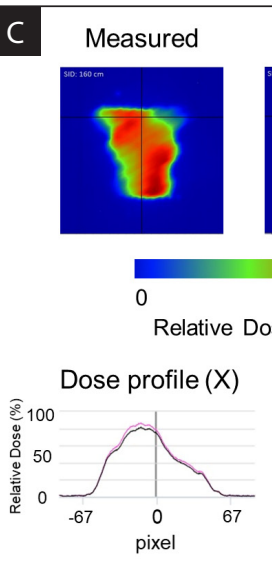

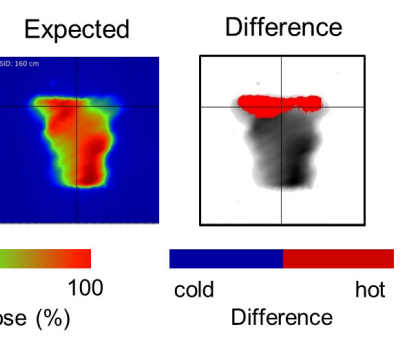

Dose profile $(Y)$

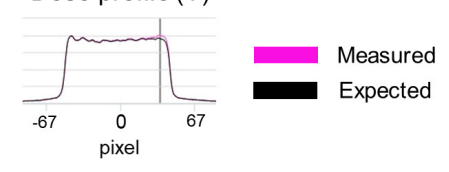

Figure 5. Example of a fraction appeared rectal gas during treatment in a clinical study (Fraction 9 in Patient 10). Sagittal view of kV images (A) before, (B) after irradiation, with PTV, bladder, and rectum contoured in red, blue, and brown. C. The result of the EPID image analyzed by DD2\% in clinical study. The EPID image of expected dose is that of Fraction 1 in Patient 9 . Dose profile $\mathrm{X}$ and $\mathrm{Y}$ are horizontal and vertical lines in the gamma index and difference 
show the cause of treatment errors identified by the EPID images. In the prostate cancer patients, the main cause of these was patient body movement and the occurrence of rectal gas during treatment. Therefore, to the best of our knowledge, this study is the first one to focus on the detectability of setup error and rectal gas during VMAT for prostate cancer patients by using EPID-based IVD.

In terms of the analysis method, previous reports suggested that the DTA component of the gamma method masked the setup error in the EPID images of individual IMRT fields [22, 25]. Our results confirmed these findings in VMAT. In the phantom study, the pass rates of gamma method in all criteria were more than $90 \%$ in a small air cavity $\left(12 \mathrm{~cm}^{3}\right)$, but those of DD of $2 \%$ and $1 \%$ were less than $90 \%$. The gamma method could not detect small changes unlike the DD method. Therefore, we used the DD method for data analysis.

Some researchers proposed that EPID-based IVD could be used to successfully detect systematic setup errors in the head region $[22,26]$. This is not in agreement with our findings because no systematic setup error was detected when simulating the anthropomorphic phantom in the range that can happen in clinical $(<5 \mathrm{~mm}$ and $<3$ degree). This is because the bone structure and body surface in the case of head cancer are more complicated than those for prostate cancer. Because the prostate is located in the center of the pelvic region, the VMAT plan is less subject to changes of the beam pass. Moreover, several reports found that systematic setup errors were detected in 3-dimensional conformal radiotherapy (3DCRT) $[15,26]$. This is because the dose distribution of the 3DCRT was sharper than that of VMAT in the edge of each field. Hence, EPID-based IVD is inadequate for systematic setup error detection in the VMAT plan for the pelvic region.

However, our study demonstrated that EPID-based IVD is useful for identifying inhomogeneity regions in the I'mRT phantom. In the pelvic region, an inhomogeneity region is highly suggestive of rectal gas. EPID-based IVD could detect small air cavities with a diameter of $2 \mathrm{~cm}$. In addition, our data indicated chronological changes in the air cavity during VMAT. Larger air cavities and a longer stop time resulted in decreased pass rates. It is suggested that EPID-based IVD is better for identifying inhomogeneity regions than detecting systematic setup errors. Moreover, the mean rectal diameter in our clinical study was $3 \mathrm{~cm}$, which was between the sizes of the large and small air cavities in the phantom studies. We decided to assess the occurrence of rectal gas for DD2\% $<90 \%$ in the clinical study by calculating the mean values of $\mathrm{T}_{75 \%}$ in the large and small air cavities on the assumption that rectal gas appears during treatment.

We measured and analyzed the EPID images in the clinical study to confirm the correlation between the pass rates and occurrence of rectal gas during VMAT. The proportion of DD $2 \%<90 \%$ was $13.4 \%$ for all the patients. Rectal gas was confirmed in $11.0 \%$ of fractions. Rosario et al. reported that the prostate position error was detected in $13.0 \%$ of fractions by using automatic detection of implanted gold seeds and the imaging application Auto Beam Hold (Varian Medical Systems) [27]. This is in agreement with our results which demonstrated the proportion of rectal-gas-positive cases and the decrease in the DD2\%. However, the proportion of $\mathrm{DD} 2 \%<90 \%$ and fractions with a rectum full of rectal gas after irradiation was only $5.8 \%$ in our study. There is a possibility that rectal gas goes in and out of the rectum during and after irradiation because there was no rectal gas before irradiation by using X-ray image registration. In addition, the proportion of $\mathrm{DD} 2 \%<90 \%$ in rectal-gas-positive cases was larger than that in rectal-gas-negative cases, but about $50 \%$ of the gas-positive cases had DD2\% $>90 \%$. This may be because rectal gas appeared from the completion of treatment to the acquisition of $\mathrm{kV}$ images. Moreover, we did not quantify changes in bladder volume in each condition because the main cause of the decline in DD2\% was the presence of inhomogeneity regions, according to the results of the phantom study. Some studies reported that EPID-based IVD had effects on the detection of rectal gas in 3DCRT for rectal and prostate cancer $[15,20]$. Woodruff et al. found that prostate cancer patients had a lower pass rate than other cancer patients for EPID-based IVD, which was due to the rectum being filled with rectal gas in CBCT scans in the last week of treatment [18]. Collectively, it is proposed that EPID-based IVD can indicate the presence of rectal gas during VMAT.

Most of the fraction in DD2\% $<90 \%$ had not the point dose over $5 \%$ dose difference, indicating that patient specific QA was acceptable in our study. This is because a large amount of rectal gas 
causes the point dose over 5\% dose difference, but we have often stopped the treatment by using CBCT images when a large amount of rectal gas was detected near the target. However, VMAT plan is steeper dose gradient and more complicated dose distribution. Therefore, the occurrence of a small amount of rectal gas near the target is imprecated in the lower target cover. Connolly et al. found that local recurrence was the greatest on the rectal side compared to other locations after radiation therapy for prostate cancer patients [28]. One reason for this may be the presence of rectal gas during treatment. It is not possible to determine the accurate dose for a target because the presence of rectal gas can cause changes in the beam pass by repeating complicated build up and down. Many reports suggest that the presence of rectal gas induces intrafractional prostate and rectum movement that prevents accurate treatment [6-11]. The presence of rectal gas is the predictor of performing accurate treatment. Therefore, it is important to identify if rectal gas is present during treatment and obtain evidence for accurate treatment. EPID-based IVD in VMAT can detect the rectal gas and monitor accurate treatment in each fraction. It is hoped that the outcome of this study will contribute to accurate radiation therapy for prostate cancer patients. One of the limitations of this study is that we were not able to compare the EPID image for the planning CT with that in each fraction. This can be used to more accurately determine the presence of rectal gas in the future.

\section{Conclusion}

This study clearly demonstrates that analysis criteria of $2 \%$ dose difference in EPID-based IVD can identify the presence of rectal gas during VMAT for prostate cancer patients. It was difficult for EPID-based IVD in VMAT for prostate cancer patients to detect systematic setup errors of the range that can happen in a clinical study.

\section{Conflicts of interest}

The authors declare that there are no conflicts of interest.

\section{Financial discrosure}

None declared.

\section{Acknowledgements}

We thank Editage (https://www.editage.jp/) for providing English language editing for our manuscript. This study was supported by JSPS KAKENHI Grant (17K15817) to Yoshihiro Ueda.

Presentation at the conference: ESTRO2019 in Milan (EP-1747)

\section{References}

1. ICRU (International Commission on Radiation Units and Measurements). Prescribing, reporting, and recording photon-beam intensity-modulated radiation therapy (IMRT) ICRU Report 83. Oxford University Press, Oxford 2010: 41-53.

2. Otto K. Volumetric modulated arc therapy: IMRT in a single gantry arc. Med Phys. 2008; 35(1): 310-317, doi: 10.1118/1.2818738, indexed in Pubmed: 18293586.

3. Hamdy FC, Donovan JL, Lane JA, et al. ProtecT Study Group. 10-Year Outcomes after Monitoring, Surgery, or Radiotherapy for Localized Prostate Cancer. N Engl J Med. 2016;375(15): 1415-1424, doi: 10.1056/NEJMoa1606220, indexed in Pubmed: 27626136.

4. Fonteyne V, Ost P, Vanpachtenbeke F, et al. Rectal toxicity after intensity modulated radiotherapy for prostate cancer: which rectal dose volume constraints should we use? Radiother Oncol. 2014; 113(3): 398-403, doi: 10.1016/j. radonc.2014.10.014, indexed in Pubmed: 25441610.

5. Ghadjar P, Thalmann GN. Comparative analysis of prostate-specific antigen free survival outcomes for patients with low, intermediate and high risk prostate cancer treatment by radical therapy. Results from the Prostate Cancer Results Study Group. BJU Int. 2012; 110(10): E431-2; author reply E432, doi: 10.1111/j.1464-410X.2012.11624.x, indexed in Pubmed: 23106765.

6. McPartlin AJ, Li XA, Kershaw LE, et al. MR-Linac consortium. MRI-guided prostate adaptive radiotherapy - A systematic review. Radiother Oncol. 2016; 119(3): 371-380, doi: 10.1016/j.radonc.2016.04.014, indexed in Pubmed: 27162159.

7. McNair HA, Wedlake L, Lips IM, et al. A systematic review: effectiveness of rectal emptying preparation in prostate cancer patients. Pract Radiat Oncol. 2014; 4(6): 437-447, doi: 10.1016/j.prro.2014.06.005, indexed in Pubmed: 25407867.

8. Padhani A, KhooV, Suckling J, et al. Evaluating the effect of rectal distension and rectal movement on prostate gland position using cine MRI. Int J Radiat Oncol Biol Phys. 1999; 44(3): 525-533, doi: 10.1016/s0360-3016(99)00040-1, indexed in Pubmed: 10348281.

9. Ogino I, Kaneko T, Suzuki R, et al. Rectal content and intrafractional prostate gland motion assessed by magnetic resonance imaging. J Radiat Res. 2011; 52(2): 199-207, doi: 10.1269/jrr.10126, indexed in Pubmed: 21436610.

10. Nichol AM, Brock KK, Lockwood GA, et al. A magnetic resonance imaging study of prostate deformation relative to implanted gold fiducial markers. Int J Radiat Oncol Biol 
Phys. 2007; 67(1): 48-56, doi: 10.1016/j.jijrobp.2006.08.021, indexed in Pubmed: 17084546.

11. Villeirs GM, De Meerleer GO, Verstraete KL, et al. Magnetic resonance assessment of prostate localization variability in intensity-modulated radiotherapy for prostate cancer. Int J Radiat Oncol Biol Phys. 2004;60(5): 1611-1621, doi: 10.1016/j. ijrobp.2004.07.711, indexed in Pubmed: 15590193.

12. Bakhtiari M, Kumaraswamy L, Bailey DW, et al. Using an EPID for patient-specific VMAT quality assurance. Med Phys. 2011; 38(3): 1366-1373, doi: 10.1118/1.3552925, indexed in Pubmed: 21520847.

13. Iori $M$, Cagni E, Paiusco $M$, et al. Dosimetric verification of IMAT delivery with a conventional EPID system and a commercial portal dose image prediction tool. Med Phys. 2010; 37(1):377-390, doi: 10.1118/1.3271107, indexed in Pubmed: 20175500.

14. Ueda Y, Miyazaki M, Nishiyama K, et al. Craniocaudal safety margin calculation based on interfractional changes in tumor motion in lung SBRT assessed with an EPID in cine mode. Int J Radiat Oncol Biol Phys. 2012; 83(3): 1064-1069, doi: 10.1016/j.ijrobp.2011.07.043, indexed in Pubmed: 22245190.

15. Kroonwijk M, Pasma K, Quint S, et al. In vivo dosimetry for prostate cancer patients using an electronic portal imaging device (EPID); demonstration of internal organ motion. Radiother Oncol. 1998; 49(2): 125-132, doi: 10.1016/s0167-8140(98)00122-4, indexed in Pubmed: 10052877.

16. Nailon WH, Welsh D, McDonald K, et al. EPID-based in vivo dosimetry using Dosimetry Check ${ }^{\mathrm{TM}}$ : Overview and clinical experience in a 5-yr study including breast, lung, prostate, and head and neck cancer patients. J Appl Clin Med Phys. 2019; 20(1): 6-16, doi: 10.1002/acm2.12441, indexed in Pubmed: 30536528.

17. Celi S, Costa E, Wessels C, et al. EPID based in vivo dosimetry system: clinical experience and results. J Appl Clin Med Phys. 2016; 17(3): 262-276, doi: 10.1120/jacmp. v17i3.6070, indexed in Pubmed: 27167283.

18. Woodruff HC, Fuangrod T, Van Uytven E, et al. First Experience With Real-Time EPID-Based Delivery Verification During IMRT and VMAT Sessions. Int J Radiat Oncol Biol Phys. 2015; 93(3): 516-522, doi: 10.1016/j.jjobp.2015.07.2271, indexed in Pubmed: 26460993.

19. Peca S, Brown DW, Smith WL. A Simple Method for 2-D In Vivo Dosimetry by Portal Imaging. Technol Cancer Res
Treat.2017;16(6):944-955, doi:10.1177/1533034617711354, indexed in Pubmed: 28585491.

20. Peca S, Sinha RS, Brown DW, et al. Portal Imaging Dosimetry Identifies Delivery Errors in Rectal Cancer Radiotherapy on the Belly Board Device. Technol Cancer Res Treat. 2017; 16(6): 956-963, doi: 10.1177/1533034617711519, indexed in Pubmed: 28585490.

21. Jomehzadeh A, Shokrani P, Mohammadi M, et al. Assessment of a $2 \mathrm{D}$ electronic portal imaging devicesbased dosimetry algorithm for pretreatment and midplane dose verification. Adv Biomed Res. 2016; 5: 171, doi: 10.4103/2277-9175.194799, indexed in Pubmed: 28028511.

22. Hsieh ES, Hansen KS, Kent MS, et al. Can a commercially available EPID dosimetry system detect small daily patient setup errors for cranial IMRT/SRS? Pract Radiat Oncol. 2017; 7(4): e283-e290, doi: 10.1016/j.prro.2016.12.005, indexed in Pubmed: 28336480.

23. ICRU (International Commission on Radiation Units and Measurements).. Prescribing, recording, and reporting photon beam therapy. ICRU Report 50. ICRU, Washington 1993: 27-8.

24. ICRU (International Commission on Radiation Units and Measurements). Prescribing, recording, and reporting photon beam therapy (Supplement to ICRU Report 50). ICRU Report 62. ICRU, Bethesda 1999: 3-11.

25. Kruse JJ. On the insensitivity of single field planar dosimetry to IMRT inaccuracies. Med Phys. 2010; 37(6): 2516-2524, doi: 10.1118/1.3425781, indexed in Pubmed: 20632563.

26. Zhuang $A H$, Olch AJ. Sensitivity study of an automated system for daily patient QA using EPID exit dose images. J Appl Clin Med Phys. 2018; 19(3): 114-124, doi: 10.1002/ acm2.12303, indexed in Pubmed: 29508529.

27. Rosario T, van der Weide L, Admiraal M, et al. Toward planning target volume margin reduction for the prostate using intrafraction motion correction with online $\mathrm{kV} \mathrm{im-}$ aging and automatic detection of implanted gold seeds. Pract Radiat Oncol. 2018; 8(6): 422-428, doi: 10.1016/j. prro.2018.04.008, indexed in Pubmed: 29907506.

28. Connolly J, Shinohara K, Presti J, et al. Local recurrence after radical prostatectomy: Characteristics in size, location, and relationship to prostate-specific antigen and surgical margins. Urology. 1996; 47(2): 225-231, doi: 10.1016/ s0090-4295(99)80421-x, indexed in Pubmed: 8607239. 